\title{
Single electron dynamics in a Hall thruster electromagnetic field profile
}

Samuel Marini, and Renato Pakter

Citation: Physics of Plasmas 24, 053507 (2017); doi: 10.1063/1.4982685

View online: https://doi.org/10.1063/1.4982685

View Table of Contents: http://aip.scitation.org/toc/php/24/5

Published by the American Institute of Physics

\section{Articles you may be interested in}

Tutorial: Physics and modeling of Hall thrusters

Journal of Applied Physics 121, 011101 (2017); 10.1063/1.4972269

Effect of anode position on the performance characteristics of a low-power cylindrical Hall thruster

Physics of Plasmas 24, 063518 (2017); 10.1063/1.4986091

Transient propagation dynamics of flowing plasmas accelerated by radio-frequency electric fields

Physics of Plasmas 24, 050703 (2017); 10.1063/1.4983059

Hall thruster microturbulence under conditions of modified electron wall emission

Physics of Plasmas 24, 053519 (2017); 10.1063/1.4984255

Effect of oblique channel on discharge characteristics of 200-W Hall thruster

Physics of Plasmas 24, 023507 (2017); 10.1063/1.4976104

Magnetic field configurations on thruster performance in accordance with ion beam characteristics in cylindrical Hall thruster plasmas

Applied Physics Letters 110, 114101 (2017); 10.1063/1.4978532

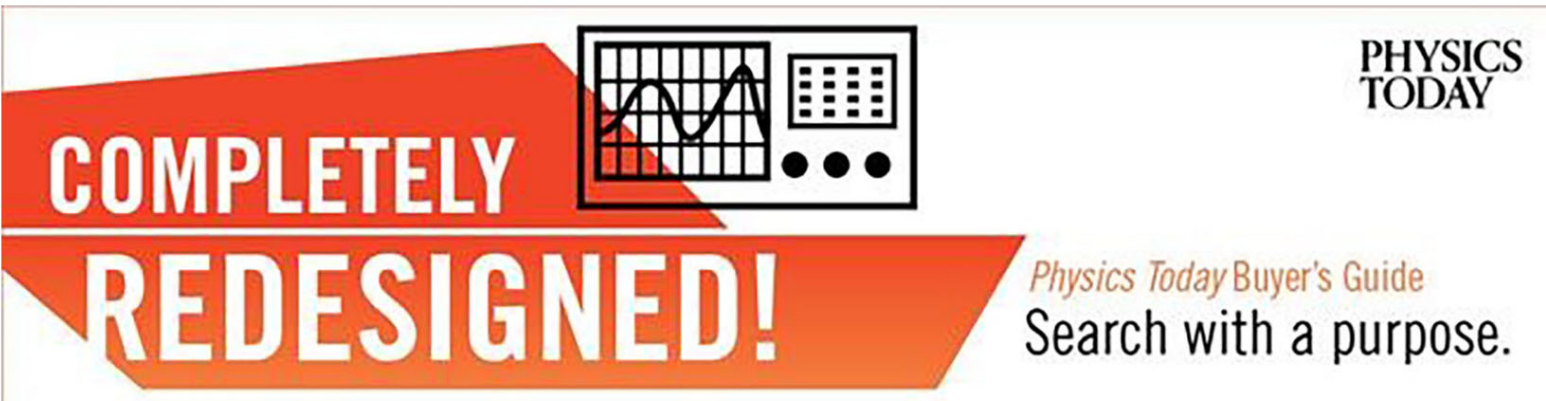




\title{
Single electron dynamics in a Hall thruster electromagnetic field profile
}

\author{
Samuel Marini ${ }^{\text {a) }}$ and Renato Pakter ${ }^{\text {b) }}$ \\ Instituto de Física, Universidade Federal do Rio Grande do Sul, Caixa Postal 15051, 91501-970 Porto Alegre, \\ RS, Brazil
}

(Received 5 January 2017; accepted 12 April 2017; published online 1 May 2017)

\begin{abstract}
In this work, the single electron dynamics in a simplified three dimensional Hall thruster model is studied. Using Hamiltonian formalism and the concept of limiting curves, one is able to determine confinement conditions for the electron in the acceleration channel. It is shown that as a given parameter of the electromagnetic field is changed, the particle trajectory may transit from regular to chaotic without affecting the confinement, which allows one to make a detailed analysis of the role played by the chaos. The ionization volume is also computed, which measures the probability of an electron to ionize background gas atoms. It is found that there is a great correlation between chaos and increased effective ionization volume. This indicates that a complex dynamical behavior may improve the device efficiency by augmenting the ionization capability of each electron, requiring an overall lower electron current. Published by AIP Publishing.

[http://dx.doi.org/10.1063/1.4982685]
\end{abstract}

\section{INTRODUCTION}

Electric propulsion has been identified as a promising technology for primary propulsion in deep-space scientific missions. ${ }^{1-3}$ Among many types of electric propulsion engines, Hall effect thrusters stand out by its current state of the art, being developed and tested for over 50 years. ${ }^{1,4}$ Recently, improvements and variations in those devices, like in the magnetic source, ${ }^{5-8}$ in the wall shaping, ${ }^{2,3}$ in the propellant gas distribution, ${ }^{9}$ and in the number of acceleration channels, ${ }^{10}$ have made long space travels using Hall thrusters closer to become a reality.

Simplistically, in a Hall thruster, electrons are accelerated and confined by crossed electric and magnetic fields ${ }^{11,12}$ in an acceleration channel. There, they collide with atoms of a propellant gas, generating ions. ${ }^{1}$ The rate of generation of ions is related to the energy of the electrons and the electronpropellant gas cross section. ${ }^{13,14}$ The ions generated in this process are electrostatically accelerated out of the acceleration channel, producing the thrust. Therefore, a key issue in order to increase the thrust power and the overall thruster efficiency is to maximize the gas ionization by the electrons in the acceleration channel. ${ }^{1-3,5-7}$

Most of the basic analyses of particle dynamics on Hall thrusters are based on models that consider a one dimensional electron flow. ${ }^{14-17}$ In such a case, the electron dynamics is integrable, being characterized by the presence of periodic orbits. ${ }^{18}$ In practice, however, the dynamics is fully three dimensional, leading to a possible break of the single particle trajectories' integrability and the onset of more complex behavior. The aim of the present paper is to investigate how such change in the electron dynamical pattern may impact the ability to ionize the background gas. In particular, we consider a simplified Hall thruster model with inhomogeneous electromagnetic fields. Using the

\footnotetext{
a)marini@ufrgs.br

b) pakter@if.ufrgs.br
}

concept of limiting curves. ${ }^{19,20}$ we are able to determine conditions that guarantee the confinement of the electron in an acceleration channel region. It is also shown that as a given parameter of the electromagnetic field is changed, the particle trajectory may transit from regular to chaotic without affecting the confinement. This allows us to make a detailed analysis of the role played by the chaos. We also compute an effective ionization volume, ${ }^{13,14}$ which measures the probability of an electron in a given trajectory to ionize background gas atoms. It is found that there is a great correlation between chaos and increased effective ionization volume. This indicates that a complex dynamical behavior tends to improve the device efficiency.

The present work is organized as follows. In Sec. II, we present the model used to describe a Hall thruster and obtain a Hamiltonian that describes the electron motion. In Sec. III, we derive a confinement criterion for the electron trajectory and determine its maximum extent along axial and radial directions as a function of the parameters of the system. In Sec. IV, we investigate the electron nonlinear dynamics and show with the aid of Poincaré maps that it may become chaotic depending on the parameters. In Sec. V, we compute the effective ionization volume for a given electron trajectory and find that it tends to increase for chaotic orbits. Our conclusions are presented in Sec. VI.

\section{MODEL}

A schematic of a Hall thruster is shown in Fig. 1. A cathode emits electrons that are forced into the acceleration channel by the electric field $\mathbf{E}_{0}$, forming a current density $\mathbf{J}_{e}$ in the negative axial direction. The radial magnetic field $\mathbf{B}_{0}$ deflects the current density $\mathbf{J}_{e}$ to the azimuthal positive direction, forming the Hall current density $\mathbf{J}_{H}$. Since the Hall current density is orthogonal to the radial magnetic field, there is also a $\mathbf{J}_{H} \times \mathbf{B}_{0}$ force that pushes the electrons along the positive axial direction. This force tends to balance the one produced by the electric field $\mathbf{E}_{0}$, creating an axial 


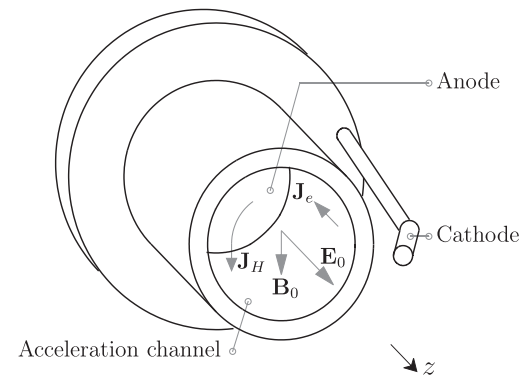

FIG. 1. Schematics of a Hall thruster. Given the symmetry of the device, it is convenient to describe the problem using cylindrical coordinates with the $z$-axis parallel to the symmetry axis of the accelerating channel.

confinement for the electron motion. Note that for simplicity, in the discussion above the current densities were defined as positive in the electron movement direction. It is also worth mentioning that this type of configuration is usually referred as cylindrical Hall thruster. ${ }^{21}$

In a real device, once the electron Hall current is established, a propellant gas, like argon, ${ }^{5,6,22}$ is inserted in the acceleration channel. The electron collisions with the initially neutral propellant gas generate positive ions, which are strongly accelerated by the electric field $\mathbf{E}_{0}$ to the axial positive direction, generating an impulse in the opposite direction. ${ }^{1,23}$ Here, however, we will focus on the electron dynamics disregarding the effects of the background gas and ions, in order to investigate features like confinement and onset of complex behavior. Physically, this corresponds to the limit of low electron current and low background gas pressure. We assume that the fields are given by

$$
\begin{gathered}
\mathbf{E}_{0}=E_{z} \hat{\mathbf{z}}, \\
\mathbf{B}_{0}=\frac{\Lambda_{r}}{r} \hat{\mathbf{r}}+B_{\theta} \hat{\theta}+B_{z} \hat{\mathbf{z}},
\end{gathered}
$$

where $E_{z}, \Lambda_{r}, B_{\theta}$, and $B_{z}$ are constant parameters which measure the axial electric field and the radial, azimuthal, and axial magnetic fields, respectively. ${ }^{24}$ It is worth noting that despite being inhomogeneous along the radial direction, $\mathbf{B}_{0}$ is divergence free, in agreement with Maxwell's equations. In a basic Hall configuration, like the one shown in Fig. 1, the magnetic field only presents a radial component which is responsible for the generation of the Hall current and axial confinement, as discussed above. However, for the sake of generality, we also consider in the model axial and azimuthal components. ${ }^{6}$ It will be shown that these components play a major role in the overall confinement and in the transition to chaotic regimes.

In a Hall thruster, the accelerating potential that generates $\mathbf{E}_{0}$ is typically much smaller than the electron rest mass. Therefore, the electron dynamics is nonrelativistic, being dictated by the Hamiltonian ${ }^{25}$

$$
H=\frac{1}{2 m}(\mathbf{P}+e \mathbf{A})^{2}-e \phi,
$$

where $m$ and $-e$ are the electron mass and charge, respectively, and $\mathbf{P}$ is the canonical momentum related to the mechanical momentum $\mathbf{p}$ by $\mathbf{p}=\mathbf{P}+e \mathbf{A}$. In Eq. (3), $\phi$ and $\mathbf{A}$ are the scalar and vector potentials, related to the electromagnetic fields by

$$
\begin{gathered}
\mathbf{E}=-\nabla \phi-\frac{\partial \mathbf{A}}{\partial t}, \\
\mathbf{B}=\nabla \times \mathbf{A} .
\end{gathered}
$$

Since the electron density in the acceleration channel is relatively low, we can safely disregard the self-fields, such that $\mathbf{E}=\mathbf{E}_{0}$ and $\mathbf{B}=\mathbf{B}_{0}$. Using Eq. (4) and the electric field described in (1), we find that the scalar potential is given by

$$
\phi(z)=-E_{z} z
$$

Here, we have chosen $\phi(z=0)=0$, where $z=0$ is the cathode axial position. Using Eq. (5) and the magnetic field described by Eq. (2), we can write the vector potential as

$$
\mathbf{A}(r, z)=\left(-\Lambda_{r} \frac{z}{r}+B_{z} \frac{r}{2}\right) \hat{\theta}+\left(-B_{\theta} r\right) \hat{\mathbf{z}} .
$$

We conveniently define dimensionless quantities $\bar{H}=H$ / $m c^{2}, \bar{P}_{r}=P_{r} / m c, \bar{P}_{z}=P_{z} / m c, \bar{P}_{\theta}=\omega_{z} P_{\theta} / m c^{2}, \bar{r}=\omega_{z} r / c$, $\bar{z}=\omega_{z} z / c, \eta_{b}=e \Lambda_{r} / m c, \eta_{e}=e E_{z} / \omega_{z} m c$, and $\eta_{p}=B_{\theta} / B_{z}$, where $c$ is the speed of light in vacuum and $\omega_{z} \equiv e B_{z} / m$. Substituting (6) and (7) in (3) the normalized Hamiltonian reads

$$
H=\frac{P_{r}^{2}}{2}+\frac{1}{2}\left(\frac{P_{\theta}}{r}-\eta_{b} \frac{z}{r}+\frac{r}{2}\right)^{2}+\frac{1}{2}\left(P_{z}-\eta_{p} r\right)^{2}+\eta_{e} z,
$$

where for simplicity, we have suppressed the bars over the dimensionless quantities. The electron dynamics satisfy $d r / d t=\partial H / \partial P_{r}, d P_{r} / d t=-\partial H / \partial r, d z / d t=\partial H / \partial P_{z}$, and $d P_{z} / d t=-\partial H / \partial z$. Because the Hamiltonian (8) does not explicitly depend on time, it is a conserved quantity along the electron trajectory. Furthermore, since there is no dependence on the angular variable $\theta$, its conjugate momentum $P_{\theta}$ is also a constant of motion. The values of these constants are determined by the initial condition. In particular, we assume that the electron is released from the cathode at $t=0$ with a vanishingly small kinetic energy, which corresponds to a vanishing initial mechanical momentum $\mathbf{p}(0)=0$. In terms of the dimensionless quantities, the mechanical momentum is given by $\mathbf{p}=P_{r} \hat{\mathbf{r}}+\left(P_{\theta} / r-\eta_{b} z / r+r / 2\right) \hat{\theta}$ $+\left(P_{z}-\eta_{p} r\right) \hat{\mathbf{z}}$. Recalling that the cathode is located at $z=0$ and defining as $r_{0}$ its radial position, we readily obtain that $\mathbf{p}(0)=0$ implies that $P_{r}(0)=0, P_{z}(0)=\eta_{p} r_{0}$, and $P_{\theta}$ $=-r_{0}^{2} / 2$. The last condition sets the value of the constant of motion $P_{\theta}$. As for the Hamiltonian, we notice that Eq. (8) can be written in terms of the mechanical momentum as $H=\mathbf{p}^{2} / 2+\eta_{e} z$. By the fact that $\mathbf{p}=0$ and $z=0$ at the cathode, we conclude that the constant value of the Hamiltonian is $H=0$. It is worth noting that in practice the electrons are released from the cathode with a thermal energy of the order of a few electron-volts. However, because this energy is much smaller than the typical accelerating energy, which is on the order of hundreds of electron-volts, to a very good approximation we can safely disregard the initial thermal energy. 


\section{CONFINEMENT CRITERION}

In order to avoid particle loss and wall heating due to electron-wall collisions, it is of utmost relevance to determine conditions for the electron confinement in the acceleration region. In this regard, perhaps a natural first step would be to perform a linear stability analysis of the fixed points in the electron dynamics. ${ }^{26}$ Nevertheless, a quick inspection on the equations of motion dictated by Hamiltonian (8) shows that in general the electron dynamics presents no fixed points at all. Therefore, we need to resort to non-linear methods to determine confinement conditions. Particularly, we take advantage of the concept of limiting curves. ${ }^{19,20}$

The procedure to obtain these curves is as follows. Isolating $z$ in the Hamiltonian (8) we can write $z=z\left(r, P_{r}\right.$, $\left.P_{z} ; H, P_{\theta}\right)$. We maximize this function with respect to the momenta by equating $\partial z / \partial P_{r}=0$ and $\partial z / \partial P_{z}=0$, which leads to $P_{r}=0$ and $P_{z}=r \eta_{p}$. Substituting these and the value of the conserved quantities $H=0$ and $P_{\theta}=-r_{0}^{2} / 2$ in $z=z\left(r, P_{r}, P_{z} ; H, P_{\theta}\right)$, we obtain

$$
z(r)=\frac{\eta_{b}\left(r^{2}-r_{0}^{2}\right)-2 \eta_{e} r^{2} \pm 2 r \sqrt{\eta_{e}^{2} r^{2}-\eta_{e} \eta_{b}\left(r^{2}-r_{0}^{2}\right)}}{2 \eta_{b}^{2}}
$$

which corresponds to the maximum excursion of $z$ as a function of $r$ that is compatible with the Hamiltonian (8) and thus represents a limiting curve in the configuration space. It is interesting to note that the limiting curve described by Eq. (9) does not depend on the parameter $\eta_{p}$. This indicates that by varying $\eta_{p}$ it is possible to change the electron dynamical behavior without modifying the confinement characteristics in the acceleration channel. From Eq. (9), we can readily obtain the maximum and the minimum values of the trajectory along the axial direction as

$$
\begin{gathered}
z_{\max }=0, \\
z_{\min }=-\frac{r_{0}^{2}}{2\left(\eta_{b}-\eta_{e}\right)} .
\end{gathered}
$$

Therefore, the electron trajectory axial span is $\left|z_{\text {min }}\right|$.

Inverting the radial and axial variables in Eq. (9), we can write the limiting curve as $r=r(z)$. From this expression, we can determine the maximum and minimum radii attained by the electron along its trajectory as

$$
\begin{gathered}
r_{\max }=r_{0} \sqrt{\frac{\eta_{b}}{\eta_{b}-\eta_{e}}}, \\
r_{\min }=0
\end{gathered}
$$

In practice, given the dimensions of a device, namely, the cathode-anode axial distance $L$ and the accelerating channel radius $R$, one can use Eqs. (11) and (12) to determine the parameters that guarantee that the electrons will not hit the cathode and the accelerating channel wall by imposing $\left|z_{\text {min }}\right|$ $<L$ and $r_{\text {max }}<R$. From Eq. (12), we note that $\eta_{b}>\eta_{e}$ must be satisfied to exist a real maximum radius. If this condition is not fulfilled, the trajectory becomes unbounded. Thus, this parameter condition guarantees the electron confinement by the electromagnetic fields. It should be stressed that in terms of the dimensional quantities the confinement condition reads $B_{z}>m E_{z} / e \Lambda_{r}$. This implies that in our model an axial component of the magnetic field is necessary in order to confine the electrons, as it introduces a radial force that balances the centrifugal force of the gyrating electron. The radial focusing introduced by the axial magnetic field is analogous to the one employed in the transport of space-charge dominated beams using solenoidal magnetic fields. ${ }^{27,28}$ It is worth noting that other field configurations take advantage of field gradients to provide radial confinement. ${ }^{1,14,17}$ Moreover, in a real device the ions in the acceleration channel may also contribute for the radial confinement of the electrons.

\section{NONLINEAR DYNAMICS AND POINCARÉ MAPS}

The electron Hamiltonian (8) describes a system with two effective degrees of freedom - the radial and the axial degrees of freedom. They are nonlinearly coupled because in general we cannot write the Hamiltonian in a separable form $H=H_{r}\left(r, P_{r}\right)+H_{z}\left(z, P_{z}\right)$. Hence, whenever the frequencies of oscillation along these two different degrees of freedom become commensurate - their ratio is equal to a rational number-a resonance and chaos are expected. ${ }^{18}$ A very convenient and powerful method to investigate the dynamics and possible onset of chaos in such system is to use Poincaré maps. ${ }^{18}$ Through the Poincaré map analysis it is possible to identify the periodic trajectories of the system and other relevant dynamic features. For an integrable trajectory, the Poincaré maps lead to closed curves called KolmogorovArnold-Moser (KAM) curves. For a non-integrable trajectory, there is a destruction of the closed orbit indicating chaos. ${ }^{18}$

To obtain a Poincaré map that represents the entire accessible phase-space, we need to consider a group of initial conditions that correspond to the same values for the constants of motion, ${ }^{29}$ namely, $H=0$ and $P_{\theta}=-r_{0}^{2} / 2$. A possible set with these characteristics is given by

$$
\begin{gathered}
r(t=0)=\alpha r_{0}, \\
P_{r}(t=0)=0, \\
z(t=0)=\frac{r_{0}^{2}\left(\alpha^{2}-1\right)}{2 \eta_{b}} \\
+\frac{\alpha r_{0}^{2}\left(-\eta_{e} \alpha+\sqrt{\eta_{e}\left(\eta_{b}+\left(\eta_{e}-\eta_{b}\right) \alpha^{2}\right)}\right)}{\eta_{b}^{2}}, \\
P_{z}(t=0)=\alpha r_{0} \eta_{p},
\end{gathered}
$$

where $\alpha$ is a real positive number that parametrizes the different initial conditions. Specifically, $\alpha=1$ corresponds to the initial condition of the real electron that is dispensed with zero kinetic energy from the cathode.

To construct the Poincaré maps, we numerically integrate the trajectories dictated by Hamiltonian (8) and plot the $P_{r} \times r$ phase space projection each time the trajectory crosses $P_{z}=0$ with $d P_{z} / d t<0$. In Fig. 2, we show the results obtained for three different values of $\eta_{p}$ and for a number of 

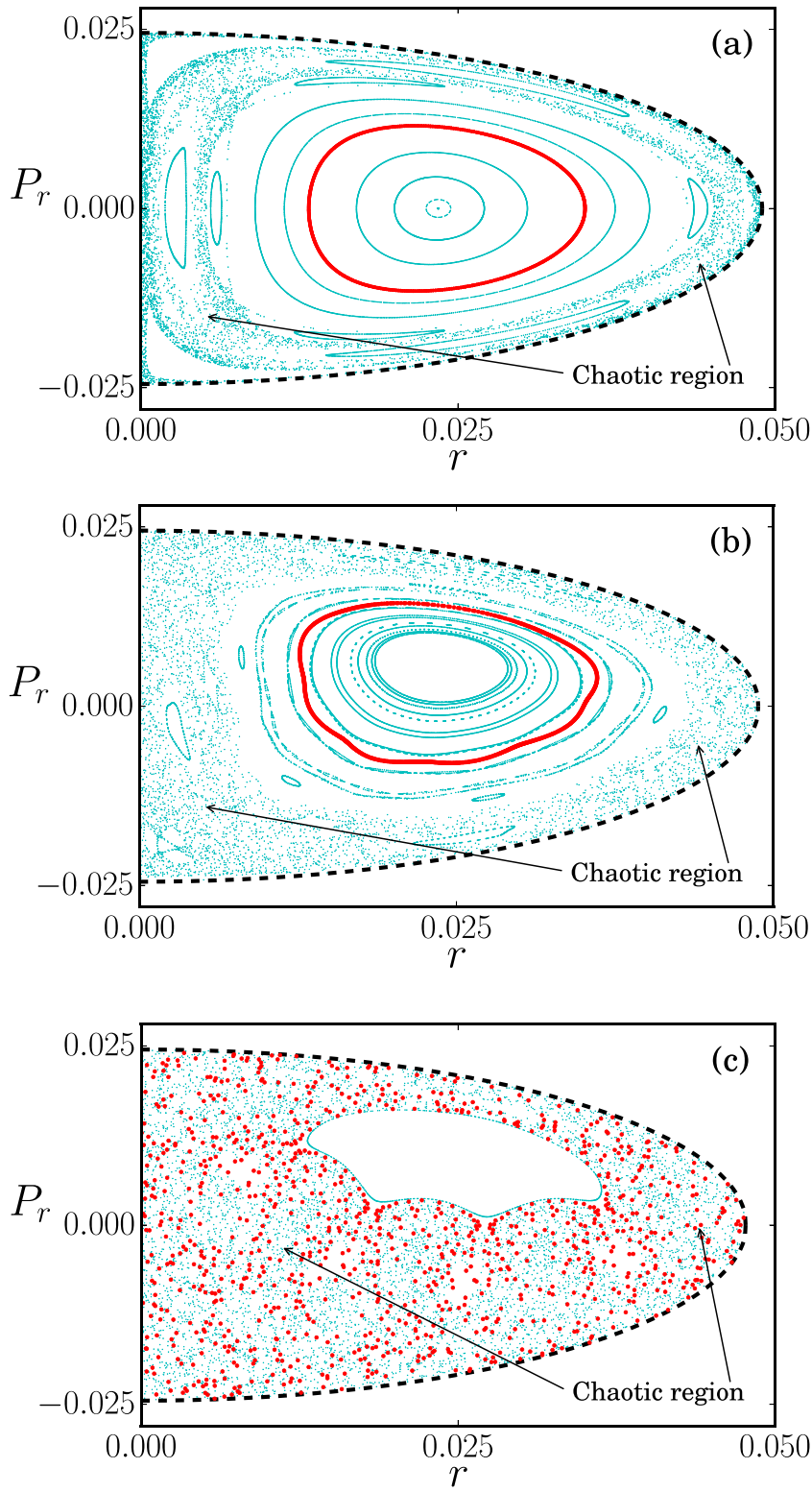

FIG. 2. Poincaré maps for the $P_{r} \times r$ phase space. The large red dots indicate the trajectory of the real electron that is ejected with vanishing energy from the cathode and the small blue dots are the trajectories obtained for other initial conditions. The dashed line is the limiting curve given by Eq. (18). The chaotic regions are indicated in the figures. The values of $\eta_{p}$ are: $\eta_{p}=0.0$ in (a), $\eta_{p}=0.04$ in (b), and $\eta_{p}=0.1163$ in (c). The remainder parameters are kept fix at $\eta_{e}=0.0120, \eta_{b}=0.0245$, and $r_{0}=0.035$.

initial conditions obtained by varying $\alpha$ in the range $0 \leq \alpha$ $\leq 1$ in Eqs. (14)-(17). The remainder parameters are kept fixed at $\eta_{e}=0.0120, \eta_{b}=0.0245$, and $r_{0}=0.035$. Note that because these parameters are not changed, all the cases presented in Fig. 2 correspond to the same confinement features and the same overall volume in the configuration space available for the electron trajectory [see Eq. (9)]. These parameters were chosen to represent a case where the accelerating potential is $V_{a}=300 \mathrm{~V}$ and the acceleration channel dimensions satisfy $L / R \approx\left|z_{\min }\right| / r_{\max }=1$. This can be readily verified using Eqs. (11) and (12) and the normalizations introduced that lead to $e V_{a} / m c^{2}=\eta_{e} r_{0}^{2} / 2\left(\eta_{b}-\eta_{e}\right)$ and $\left|z_{\min }\right| / r_{\max }=r_{0} / 2 \sqrt{\eta_{b}\left(\eta_{b}-\eta_{e}\right)}$, where $L=\left|z_{\min }\right|$ has been assumed. In the figure, the larger red dots are the results for the trajectory with $\alpha=1$, i.e., for the electron dispensed with zero kinetic energy from the cathode. The smaller blue dots correspond to initial conditions with varying $\alpha$ values (different from unity). In all the panels of Fig. 2 we see that the Poincaré maps only occupy a portion of the $P_{r} \times r$ space. The occupied region is bounded by a $P_{r}(r)$ limiting curve that can be derived by using the prescribed values for the constants of motion, $H=0$ and $P_{\theta}=-r_{0}^{2} / 2$, and the plotting condition $P_{r}=0$ in Eq. (8). This leads to

$$
P_{r}= \pm \frac{\sqrt{\left(\eta_{e}^{2}-\eta_{b}^{2} \eta_{p}^{2}\right) r^{2}-\eta_{b} \eta_{e}\left(r^{2}-r_{0}^{2}\right)}}{\eta_{b}}
$$

which is represented in the figure by the dashed curves. Panel (a), for $\eta_{p}=0.0$, shows a phase space that is predominantly regular with the occurrence of many KAM curves. There is only a limited chaotic region near the boundary of the forbidden area. In particular, the trajectory of the true electron is regular and, as a consequence, tend to explore very little of the available phase space. As $\eta_{p}$ is increased to $\eta_{p}=0.04$, the phase space becomes more distorted-it is not symmetric with respect to the $P_{r}=0$ line anymore - and the chaotic region tends to enlarge, as shown in panel (b). Despite the enlarged chaotic region, the true trajectory is still regular and restricted to a small portion of the available phase space. We also notice the presence of a blank region centered near $r=0.025$ and $P_{r}=0.006$. Although this region lies within the limiting curve, it is not accessed by trajectories with the prescribed initial condition and, in particular, by the true electron. If we keep increasing $\eta_{p}$, as in panel (c) with $\eta_{p}=0.1163$, we notice that the true trajectory is finally arrested by the chaotic region and starts to explore a much larger area in the phase space. Hence, we see that even when the confinement condition and the total volume in the configuration space accessible to the particle are the same, the effective area explored may largely increase as the trajectory becomes chaotic.

\section{CHAOS AND IONIZATION CROSS SECTION}

As mentioned in Introduction, a key issue in order to increase the thruster efficiency is to maximize the gas ionization by the electrons in the acceleration channel. In Sec. IV, we have shown that chaos can largely increase the effective volume swept by the electron in phase space. Therefore, a natural question that arises is: can chaos improve the background gas ionization by the electrons?

In order to answer this question, we need to calculate for which parameters the electron is more likely to ionize the background gas. At any given point in its trajectory, the probability of the electron ionizing a background atom is measured by the ionization cross section $\sigma$, which depends on the electron kinetic energy $\varepsilon$ and the particular gas that is being used. Multiplying the cross section by the electron instantaneous displacement $d l$, we obtain an effective ionization volume for the electron trajectory, given by $d V_{i}=\sigma(\varepsilon) d l$. Since the magnetic field does no work on the electron, its kinetic energy is completely determined by the 
scalar potential as $\varepsilon=-e \phi$. Using (6) and dimensionless quantities we find that

$$
\varepsilon=-\eta_{e} z
$$

Hence, we can express the ionization cross section in terms of the axial coordinate, $\sigma(z)$. Moreover, we can write $d l=v d t$, where $v$ is the electron speed which can be written in terms of the kinetic energy and, consequently, of the axial coordinate as $v=\left(-2 \eta_{e} z\right)^{1 / 2}$. Taking all this into account, the total effective ionization volume can be obtained by integrating

$$
\frac{d V_{i}}{d t}=\left(-2 \eta_{e} z\right)^{1 / 2} \sigma(z)
$$

along the electron trajectory. The larger $V_{i}$, the more likely is the electron to ionize background gas atoms.

In Fig. 3, we show the results for the total effective ionization volume (dashed curve) as $\eta_{p}$ is varied. The results are obtained by considering an accelerating potential of $300 \mathrm{~V}$ and the first ionization cross section $\sigma$ for the argon. ${ }^{13}$ The orbits were integrated along a fixed time interval which is much larger than the typical oscillating period of the electron in the accelerating channel. To simplify the comparison, we normalize $V_{i}$ to its lowest value in the range, $V_{\min }$. To compare these results with the onset or not of chaos, we also compute the largest Lyapunov exponent $\lambda$ (Refs. 18 and 30) for the electron trajectory which gives a direct indication if the trajectory is regular or chaotic for the given parameters. The Lyapunov exponent is a measure of how fast the distance between neighboring initial conditions increase in phase space as time evolves. It is known that when the system is chaotic it presents a high sensitivity to initial conditions, such that two orbits that are infinitely close at $t=0$ tend to diverge exponentially with their distance scaling as $e^{\lambda t}$. We use the method developed in Ref. 30 to numerically calculate the largest Lyapunov exponent, such that when $\lambda$ is

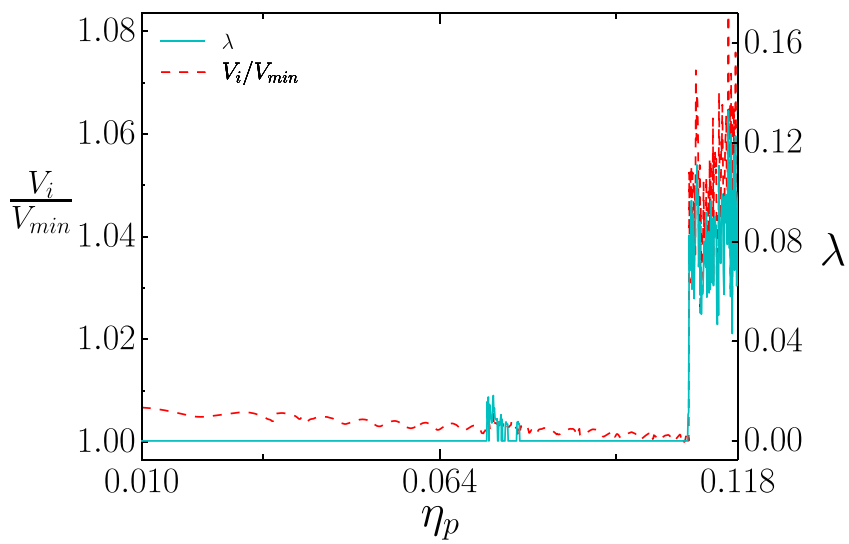

FIG. 3. Normalized ionization volume, $V_{i} / V_{\min }$ (red dashed curve), and Lyapunov exponent, $\lambda$ (blue solid curve), as a function of $\eta_{p} . V_{\min }$ is the lowest value of $V_{i}$ in the interval. The remainder parameters are the same as in Fig. 2. The scale on the left (right) axis refers to $V_{i} / V_{\min }(\lambda)$. The range of variation of the parameter $\alpha_{p}$, namely $0.010 \leq \eta_{p} \leq 0.0118$, was chosen to stress the transition from regular to chaotic trajectory that occurs in the vicinity of $\eta_{p}=0.118$. Note that this transition is accompanied by a clear increase in the effective ionization volume. positive, the trajectory is chaotic; when $\lambda$ is zero, the trajectory is regular. The results for $\lambda$ are represented by the solid curve in Fig. 3. We see that there is a great correlation between chaos and increased effective ionization volume. For the particular parameters used, an increase of up to $8 \%$ in $V_{i}$ is found when the trajectory becomes chaotic. This improvement may have an important impact on the device efficiency. ${ }^{3,5,9}$

In order to investigate in more detail the role of chaos and its effect on the ionization, we select from Fig. 3 two sets of parameters that lead to completely distinct results. In Fig. 4, we show results for $\eta_{p}=0.0879$, which corresponds to $V_{i}=V_{\text {min }}$ and a regular (non chaotic) electron orbit. In panel (a), it is shown the quasiperiodic electron trajectory in the configuration space (solid curve). The limiting curve (dashed line) of Eq. (9) is also shown. It is clear that the trajectory is indeed bounded by the limiting curve and that it does not visit a good portion of the available space. In panel (b), the probability distribution $n(z)$ (histogram) along the axial axis for the electron trajectory is presented. It presents two peaks located in the vicinity of the turning points of the orbit, where the velocity in $z$ vanishes and the particle spends a good amount of time. Typically, one can expect that any regular orbit will present such peaks because the location of the turning points along a given direction is relatively well defined. To analyze how the distribution affects the

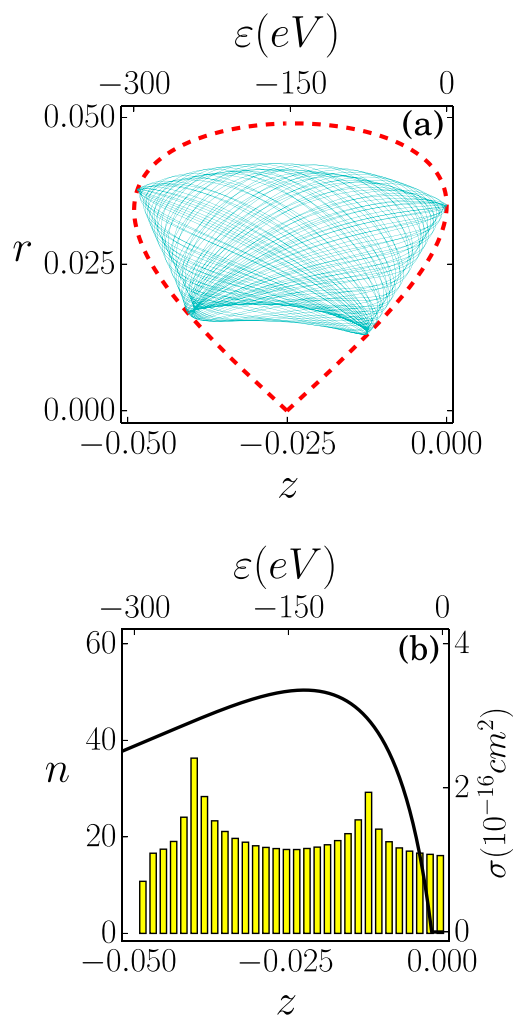

FIG. 4. Panel (a) displays the electron trajectory (solid curve) in the configuration space $(r \times z)$ and the corresponding limiting curve (dashed curve) described by Eq. (9). Panel (b) shows the cross-section $\sigma$ (solid curve) and the probability distribution $n$ (histogram) as a function of axial coordinate $z$ at bottom and as a function of energy $\varepsilon$ at top. Note that in our model there is a direct relation between $\varepsilon$ and $z$ given by Eq. (19). Here, $\eta_{p}=0.0879$ which leads to $\lambda=0.0$ and $V_{i} / V_{\text {min }} \approx 1.0$ (see Fig. 3). 
ionization, we also show in panel (b) the corresponding ionization cross section $\sigma(z)$ (solid line). It is not difficult to figure out why this particular case leads to a relatively small effective ionization volume: none of the peaks of $n(z)$ match the maximum of $\sigma(z)$. Even if one could perfectly tune the parameters such that one of the peaks of $n(z)$ matched the maximum of $\sigma(z)$, the electron would still spend a good amount of time near the other peak with a lower ionization cross section and the increase in the total ionization volume would be modest. This is exactly what Fig. 3 shows us: as long as the trajectory is regular there are only slight variations in $V_{i}$.

In Fig. 5, we consider the case $\eta_{p}=0.1163$, which corresponds to a chaotic trajectory that leads to the maximum value of $V_{i}$ in Fig. 3. In Fig. 5(a) we notice again that the trajectory is well contained in the limiting curve. However, differently from the regular trajectory of Fig. 4(a), now the orbit explores basically all its accessible region due to the chaotic diffusion. Also different from the regular case, the probability distribution $n(z)$ for the chaotic orbit shown in Fig. 5(b) presents roughly a single peak. Tuning the parameters to coincide this peak with the maximum of $\sigma(z)$ leads to the enhanced total effective ionization volume observed.

In our model, we assume that the electron interacts with static electromagnetic fields. However, in a real device there
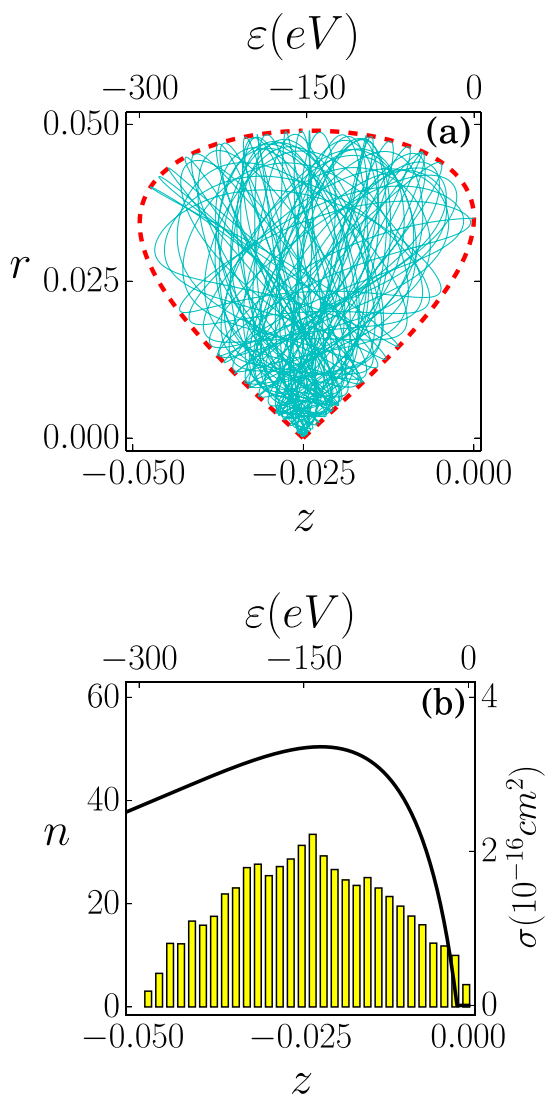

FIG. 5. Panel (a) displays the electron trajectory (solid curve) in the configuration space $(r \times z)$ and the corresponding limiting curve (dashed curve) described by Eq. (9). Panel (b) shows the cross-section $\sigma$ (solid curve) and the probability distribution $n$ (histogram) as a function of axial coordinate $z$ at bottom and as a function of energy $\varepsilon$ at top. Note that in our model there is a direct relation between $\varepsilon$ and $z$ given by Eq. (19). Here, $\eta_{p}=0.1163$ which leads to $\lambda=0.125$ and $V_{i} / V_{\min }=1.0825$ (see Fig. 3). may be instabilities that cause field oscillations at different frequencies. In particular, plasma instabilities occur in the MHz range $\mathrm{e}^{31,32}$ and ionization oscillations cause variations in the $\mathrm{kHz}$ range. ${ }^{33}$ It is therefore interesting to compare these timescales to the typical ones found in our model for the electron period and the onset of chaos. For that, let us consider the case presented in Fig. 5 and assume that the axial magnetic field-which determines the time normalization-is of the order of $B_{z} \sim 10^{-2} \mathrm{~T}^{6}{ }^{6}$ From the simulation, we find that the electron trajectory period of oscillation $T_{o}$ is around 6.0 in terms of the normalized time. This corresponds to $T_{o}=3.4 \mathrm{~ns}$, which is much smaller than the typical period of the afore mentioned instabilities. Hence, from the point of view of nonlinear dynamics, their effect on the electron trajectory is expected to be small since they are far from being in resonance. As for the chaos onset time scale, we use the Lyapunov exponent to estimate it. ${ }^{18}$ When the distance between initially neighboring initial conditions increases by a large factor, let us say $e^{\lambda t} \sim 100$, we expect noticeable chaotic effects. Taking into account that $\lambda=0.125$ for the example presented in Fig. 5, this corresponds to times on the order of $T_{\text {chaos }} \sim 10^{-7} \mathrm{~s}$, which are still on the same order of magnitude of the fastest oscillations caused by plasma instabilities. In the model, we also disregard the effects of the background gas and ions, as well as the self-interaction among the electrons. These assumptions are valid when the ionization ratio and the space charge forces are sufficiently small. Particularly, if we want the electron trajectory to fully exhibit its chaotic properties without being much affected by ionization, we must impose that $n_{g} V_{i}\left(T_{\text {chaos }}\right) \ll 1$, where $n_{g}$ is the background gas density and $V_{i}\left(T_{\text {chaos }}\right)$ represents the effective ionization volume computed at a time $T_{\text {chaos }}$ such that the chaotic effects are noticeable. Using $T_{\text {chaos }}$ computed above and $V_{i}\left(T_{\text {chaos }}\right)$ obtained from the simulation, this condition leads to $n_{g} \ll 10^{22} \mathrm{~m}^{-3}$. Regarding the space charge, we can estimate the self electric field $E_{s}$ by assuming that the electrons are uniformly distributed in the cylindrical volume of the accelerating channel and calculate its value along the axis at $z=0$. One can safely disregard the space charge effects as long as the electron density $n_{e}$ is small enough such that $E_{s} \ll E_{z}$. Using the parameters of Fig. 5, this corresponds to $n_{e} \ll 5 \times 10^{15} \mathrm{~m}^{-3}$. It should be noted that in practice the ions screen part of the self electric field and the above condition may be somewhat relaxed.

To test the correlation between chaotic orbits and enhanced ionization for a broader range of cases, we consider the parameter space plots shown in Fig. 6. In these plots, we vary both $\eta_{b}$ and $\eta_{p}$. In panel (a), we show the largest Lyapunov exponent $\lambda$. The white (dark) regions correspond to regular (chaotic) trajectories with $\lambda=0(\lambda>0)$. We note that the chaotic regions are distributed in the parameter space in a complex, nontrivial way. In panel (b), we present the results obtained for the total effective ionization volume. The darker is the region the more enhanced is the ionization. Comparing panels (a) and (b), it is clear that there is a great correlation between improved ionization and chaos.

We also performed an analogous analysis considering different accelerating potentials and different acceleration channel aspect ratios $L / R$. In agreement with the results 

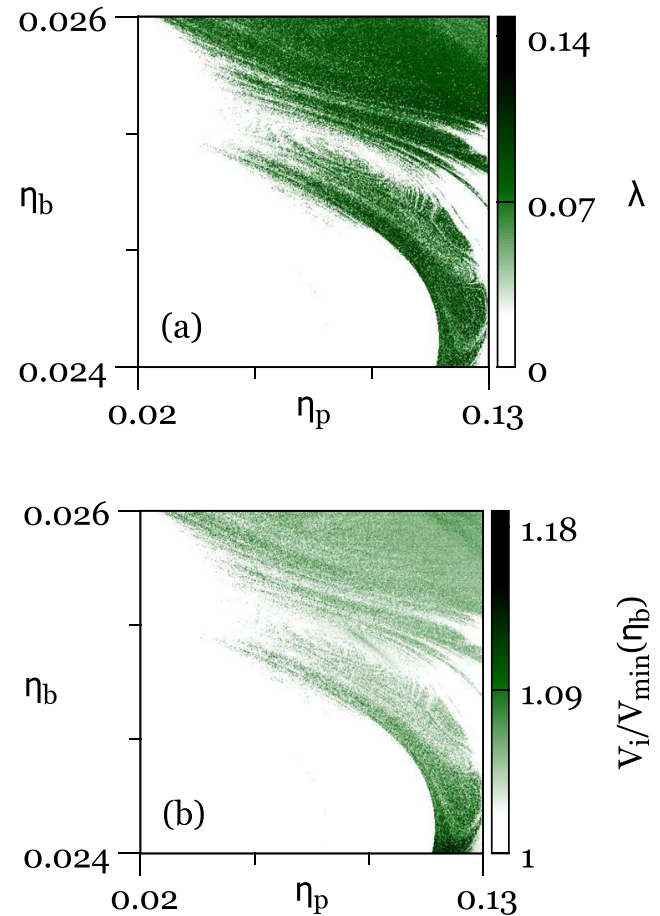

FIG. 6. Panel (a) displays the Lyapunov exponent as a function of the parameters $\eta_{b}$ and $\eta_{p}$. White regions indicate regular dynamics, while the green-black regions indicate chaotic dynamics. Panel (b) displays the normalized ionization volume as a function of the parameters $\eta_{b}$ and $\eta_{p}$. The darker is the region, the larger is $V_{i} / V_{\min }\left(\eta_{b}\right) . V_{\min }\left(\eta_{b}\right)$ corresponds to the lowest value of $V_{i}$ in the interval of variation of $\eta_{p}$ for a given $\eta_{b}$.

presented, we always find a great correlation between chaos and improved ionization. Moreover, we also found that typically the larger is the accelerating potential, the larger is the relative improvement that one can achieve by considering chaotic trajectories.

\section{CONCLUSIONS}

In the present paper, we have investigated how chaotic electron trajectories may affect the ability to ionize the background gas in a Hall thruster system. We considered a simplified three dimensional model with inhomogeneous electromagnetic fields. Using a Hamiltonian formalism and the concept of limiting curves, ${ }^{19,20}$ we were able to determine conditions that guarantee the confinement of the electron in the acceleration channel. It was also shown that as a given parameter of the electromagnetic field is changed, the particle trajectory could transit from regular to chaotic without affecting the confinement. This allowed us to make a detailed analysis of the role played by the chaos and to relate it to the ionization volume, which measures the probability of an electron in a given trajectory to ionize background gas atoms. It was found that there is a great correlation between chaos and increased effective ionization volume. This indicates that a complex dynamical behavior tends to improve the device efficiency. The chaotic electron dynamics discussed here may be an important ingredient to explain the anomalous transport that tends to enhance the cross field electron mobility in Hall thrusters. ${ }^{34}$ In real devices, the field configuration is generally more involved than the one considered in our model. The method employed here to analyze the electron trajectory can be extended to such field configurations, leading to a more complex set of limiting curves and a richer parameter space dependence concerning the onset of chaos. Nevertheless, it is anticipated that the beneficial effects generated by chaotic electron trajectories on their ability to ionize background gas atoms is a general phenomena, valid for any field configuration.

\section{ACKNOWLEDGMENTS}

This work was supported by $\mathrm{CNPq}$, Brazil, and by the Air Force Office of Scientific Research (AFOSR), USA, under the Grant No. FA9550-16-1-0280. The authors are grateful to Felipe B. Rizzato and Eduardo A. Peter for valuable discussions.

${ }^{1}$ D. M. Goebel and I. Katz, Fundamentals of Electric Propulsion (Wiley, Hoboken, 2008).

${ }^{2}$ J. Vaudolon, S. Mazouffre, C. Hénaux, D. Harribey, and A. Rossi, Appl. Phys. Lett. 107, 174103 (2015)

${ }^{3}$ S. Mazouffre, S. Tsikata, and J. Vaudolon, J. Appl. Phys. 116, 243302 (2014).

${ }^{4}$ E. Y. Choueiri, J. Propul. Power 20, 193 (2004).

${ }^{5}$ J. L. Ferreira, A. A. Martins, R. Miranda, A. B. Schelin, L. de Souza Alves, E. G. Costa, H. O. Coelho, A. C. B. Serra, and F. Nathan, Comput. Appl. Math. 35, 711 (2016).

${ }^{6}$ J. L. Ferreira, J. H. C. de Souza, I. da Silveira Rego, and I. S. Ferreira, in Proceedings of the 12th International Congress on a Plasma Physics (2004).

${ }^{7}$ S. Mazouffre, G. Bourgeois, K. Dannenmayer, and A. Lejeune, J. Phys. D: Appl. Phys. 45, 185203 (2012)

${ }^{8}$ Y. Raitses, E. Merino, and N. J. Fisch, J. Appl. Phys. 108, 093307 (2010).

${ }^{9}$ Y. Gao, H. Liu, P. Hu, H. Huang, and D. Yu, Phys. Plasmas 23, 083514 (2016).

${ }^{10}$ S. J. Hall, R. E. Florenz, A. Gallimore, H. Kamhawi, D. L. Brown, J. E. Polk, D. M. Goebel, and R. R. Hofer, in 50th AIAA/ASME/SAE/ASEE Joint Propulsion Conference, Propulsion and Energy Forum (2014).

${ }^{11}$ S. Marini, F. B. Rizzato, and R. Pakter, Phys. Plasmas 23, 033107 (2016).

${ }^{12}$ S. Marini, F. B. Rizzato, and R. Pakter, Phys. Plasmas 21, 083111 (2014).

${ }^{13}$ P. L. Bartlett and A. T. Stelbovics, Phys. Rev. A 66, 012707 (2002).

${ }^{14}$ E. Ahedo, P. Martínez-Cerezo, and M. Martínez-Sánchez, Phys. Plasmas 8, 3058 (2001).

${ }^{15}$ E. Y. Choueiri, Phys. Plasmas 8, 1411 (2001).

${ }^{16}$ K. Hara, I. D. Boyd, and V. I. Kolobov, Phys. Plasmas 19, 113508 (2012).

${ }^{17}$ K. Kwon, M. L. R. Walker, and D. N. Mavris, Plasma Sources Sci. Technol. 20, 045021 (2011).

${ }^{18}$ A. J. Lichtenberg and M. A. Lieberman, Regular and Chaotic Dynamics, 2nd ed. (Springer-Verlag, 1992).

${ }^{19}$ M. Frichembruder, R. Pakter, G. Gerhardt, and F. B. Rizzato, Phys. Rev. E 62, 7861 (2000)

${ }^{20}$ E. E. Zotos, Mon. Not. R. Astron. Soc. 446, 770 (2015).

${ }^{21}$ Y. Raitses and N. J. Fisch, Phys. Plasmas 8, 2579 (2001).

${ }^{22}$ D. Ichihara, A. Uchigashima, A. Iwakawa, and A. Sasoh, Appl. Phys. Lett. 109, 053901 (2016).

${ }^{23}$ S. T. Thornton and J. B. Marion, Classical Dynamics of Particles and Systems, 5th ed. (Cengage Learning India, 2012).

${ }^{24}$ L. B. King, in 29th International Electric Propulsion Conference (2005)

${ }^{25} \mathrm{H}$. Goldstein, C. Poole, and J. Safko, Classical Mechanics, 3rd ed. (Addison Wesley, 2001).

${ }^{26} \mathrm{~S}$. H. Strogatz, Nonlinear Dynamics and Chaos: With Applications to Physics, Biology, Chemistry, and Engineering (Westview Press, 2014).

${ }^{27}$ R. C. Davidson and H. Qin, Physics of Intense Charged Particle Beams in High Energy Accelerators (World Scientific, Singapore, 2001).

${ }^{28}$ Y. Levin, R. Pakter, and T. N. Teles, Phys. Rev. Lett. 100, 040604 (2008).

${ }^{29}$ R. Pakter, S. R. Lopes, and R. L. Viana, Physica D 110, 277 (1997). 
${ }^{30}$ A. Wolf, J. B. Swift, H. L. Swinney, and J. A. Vastano, Physica D 16, 285 (1985).

${ }^{31}$ M. D. Campanell, A. V. Khrabrov, and I. D. Kaganovich, Phys. Rev. Lett. 108, 235001 (2012).

${ }^{32}$ T. Lafleur, S. D. Baalrud, and P. Chabert, Phys. Plasmas 23, 053503 (2016).
${ }^{33}$ K. Hara, M. J. Sekerak, I. D. Boyd, and A. D. Gallimore, J. Appl. Phys. 115, 203304 (2014).

${ }^{34}$ R. Hofer, I. Katz, D. Goebel, K. Jameson, R. Sullivan, L. Johnson, and I. Mikellides, in 44th AIAA/ASME/SAE/ASEE Joint Propulsion Conference and Exhibit (2008). 